\title{
Chest radiographic staging in allergic bronchopulmonary aspergillosis: relationship with immunological findings
}

\author{
J.L. Kiely*, L. Spense**, M. Henry*, M.F. Hurley**, N. Kelleher*, C.P. Bredin*
}

Chest radiographic staging in allergic bronchopulmonary aspergillosis: relationship with immunological findings. J.L. Kiely, L. Spense, M. Henry, M.F. Hurley, N. Kelleher, C.P. Bredin. (OERS Journals Ltd 1998.

ABSTRACT: The question of whether a chest radiographic severity staging system could be correlated with standard blood/serum diagnostic indices in allergic bronchopulmonary aspergillosis (ABPA) was addressed in 41 patients.

Asthma and positive Aspergillus fumigatus (AF) serology were considered essential diagnostic inclusion criteria. Eosinophil count, serum immunoglobulin (Ig)E and immediate skin hypersensitivity were also tested to grade patients as "definite" or "likely" ABPA. Definite cases had all five of these factors present, whereas likely cases had three or more. Chest radiographs were examined by experienced radiologists blinded to the clinical data. The six-stage radiographic score (0-5) was based on the severity and duration of changes seen: stage 0: normal; stage 1: transient hyperinflation; stage 2: transient minor changes; stage 3: transient major changes; stage 4: permanent minor changes; and stage 5: permanent major changes.

Significant positive correlations $(\mathbf{p}<0.05)$ were observed between peak AF titres (expressed as an index), peak eosinophil count and radiographic severity stage. When considered as subgroups, these correlations approached, but did not reach, significance for the group with "likely" ABPA $(n=28)$, but in the group with definite ABPA $(n=13)$, there was a high correlation between radiographic score and peak AF index $(r=0.59)$, as well as peak eosinophil count $(r=0.62)$.

This study suggests that the peak Aspergillus fumigatus index and eosinophil counts correlate best with the severity of radiographic stages in allergic bronchopulmonary aspergillosis. This chest radiographic staging system may be useful in the clinical assessment and management of patients with allergic bronchopulmonary aspergillosis, particularly in those patients with more severe radiographic stages.

Eur Respir J 1998; 12: 453-456.
Depts of *Respiratory Medicine, **Radiology and Immunology, Cork University Hospital, Wilton, Cork, Ireland.

Correspondence: C.P. Bredin

Dept of Respiratory Medicine

Cork University Hospital

Wilton

Cork

Ireland

Fax: 35321343307

Keywords: Aspergillosis immunology

radiographic staging

Received: July 241997

Accepted after revision: April 141998
Following the initial description of allergic bronchopulmonary aspergillosis (ABPA) by Hinson et al. [1] in 1952 in the UK, this condition has since been reported worldwide [2]. Fungi other than Aspergillus fumigatus (AF) have also been identified as causing a similar clinicopathological process [3, 4]. HENDERSON et al. [5], in a study of 107 consecutive patients admitted to a hospital for chronic chest disease, reported $22 \%$ of 46 asthmatics to have ABPA. Another study indicated that the prevalence period $>4$ yrs of allergic bronchopulmonary mycosis in a hospital outpatient respiratory medicine department was just $>1 \%$ [6]. There is still an incomplete agreement as to what clinical, radiographic, and laboratory features are necessary to diagnose ABPA, but Rosenberg et al. [7] and SAFRSTEIN et al. [8] are in broad agreement and recommend six of the following "primary criteria" for definite diagnosis: episo-dic bronchial obstruction (asthma), peripheral blood eosinophilia, immediate skin reactivity to aspergillus antigen, precipitating antibodies against aspergillus antigen, elevated serum immunoglobulin $(\mathrm{Ig}) \mathrm{E}$, history of pulmonary infiltrates (transient or fixed) and central bronchiectasis. In pulmonary sarcoidosis, chest radiographic severity stag-ing (stages 1-4) has been used as an index of activity of this disease and of prognosis [9]. A clinical staging system has been suggested for use in ABPA [10]. The question was addressed as to whether a chest radiographic staging system in ABPA could be related to blood/serum diagnostic criteria, i.e. eosinophil count, serum $\operatorname{IgE}$ and aspergillus antibodies. If a correlation to these criteria was found it was proposed that chest radiographic staging may be a useful monitor of disease activity and possibly of prognosis, analagous to the use of chest radiographic staging in pulmonary sarcoidosis.

\section{Patients and methods}

Patients selected for inclusion in this study attended the Respiratory Medicine clinic at the University Hospital (Cork, Ireland) during the 11 yrs 1985-1995. There was a diagnosis of asthma in all cases. Patient details are listed in table 1 and all five of the nonradiological criteria proposed by Rosenberg et al. [7] and SAFRSTEIn et al. [8] were used 
Table 1. - Patient characteristics

\begin{tabular}{|c|c|c|c|c|}
\hline & $\begin{array}{l}\text { Age } \\
\text { yrs }\end{array}$ & $\begin{array}{l}\text { Aspergillus } \\
\text { index }\end{array}$ & $\begin{array}{l}\text { IgE level } \\
\mathrm{IU} \cdot \mathrm{mL}^{-1}\end{array}$ & $\begin{array}{l}\text { Eosinophil } \\
\text { count } \\
\text { cells } \cdot \mu \mathrm{L}^{-1}\end{array}$ \\
\hline $\begin{array}{l}\text { Whole group } \\
(\mathrm{n}=41)\end{array}$ & $\begin{array}{c}47.71 \\
(19.46)\end{array}$ & 4.15 & 820.00 & 580 \\
\hline $\begin{array}{l}\text { "Definite" } \\
\text { ABPA }(n=13)\end{array}$ & $\begin{array}{c}47.92 \\
(17.20)\end{array}$ & 3.4 & 1000 & 780 \\
\hline $\begin{array}{l}\text { "Likely" ABPA } \\
(\mathrm{n}=28)\end{array}$ & $\begin{array}{l}45.16 \\
(20.30)\end{array}$ & 5.05 & 798.50 & 530 \\
\hline
\end{tabular}

Age is given as mean (SD); other data are presented as median values. Laboratory reference range for Aspergillus fumigatus index; positive $>2.5$; reference range for immunoglobulin $(\mathrm{Ig}) \mathrm{E}$ upper limit of normal=150 IU.mL $\mathrm{mL}^{-1}$; upper limit for eosinophils $=450$ cells $\cdot \mu \mathrm{L}^{-1}$. ABPA: allergic bronchopulmonary aspergillosis.

in patient selection. All patients had positive AF serology. Cases negative for AF serology were excluded. The presence of peripheral blood eosinophilia, positive immediate skin reactivity to prick testing with $\mathrm{AF}$ antigen and elevated IgE were used to classify patients as "definite" (all five of the above criteria) or "likely" (at least one of the latter three criteria present). Patients with positive AF serology and asthma but no other criteria were ex-cluded. Patients were also excluded if their radiographs were unavailable. Chest radiographic changes were not taken into consideration by the clinicians when evaluating the patient's data for categorization and the radiographs used for grading were not necessarily synchronous with the peak blood/serum samples. This method was chosen because permanent chest radiographic changes in the higher grades, as outlined below, do not revert to lower grades and treatment will alter blood/serum indices [10]. All of the patients' chest radiographs were reviewed by two experienced radiologists who were blinded to the patient's category, i.e. "definite ABPA", "likely" ABPA or "excluded". A six-scale scoring method was used: Grade 0 indicated a normal radiograph, Grade 1 was assigned to transient hyperinflation without other changes, Grade 2 reflected transient minor changes such as minor infiltrate, small nodules and, tramline shadows, and Grade 3 indicated transient major changes such as "gloved finger" or band shadows, major infiltrates and consolidation, transient atelectasis or volume loss. "Transient" in each of these grades indicates $<6$ months duration. Grade 4 was identified by permanent ( $>6$ months) minor changes such as small nodules, ring shadows, "hair" or parallel lines, while Grade 5 was indicated by permanent major changes such as lobar consolidation or collapse, fixed infiltrates or localized fibrosis, atelectasis, lobar shrinkage or proximal bronchiectasis. The highest grade reported was used for correlation with blood/serological data.

Antibodies to AF were measured by enzyme-linked immunosorbent assay (ELISA). Somatic and culture filtrate antigens of AF (Mercia Diagnostics, Dublin, Ireland) were used in this process. Alkaline phosphatase-conjugated antihuman IgG (Boehringer, Dublin, Ireland) and p-nitrophenylophosphate (Sigma, Dorset, UK) were used in the secondary reaction. Tests were performed using a microtitre reader (Minireader MR 590; Shaw Scientific, Dublin, Ireland). Internal positive controls (positively react- ing sera) were standardized against commercially obtained positive controls (Mercia Diagnostics).

Results of peak AF antibody titres were expressed as an index or ratio. Positively reacting sera gave readings that were at least 2.5 times that of negative controls. Peak eosinophil counts were also used. Total serum IgE was measured by a solid-phase immunoassay (Unicap Allergy System, Pharmacia, Dublin, Ireland).

\section{Statistical analysis}

The data for chest radiographs grade, peak aspergillus indices and peak eosinophil counts were not normally distributed; therefore, correlations were determined using Spearman's rank order method. Analysis of total serum IgE data included log transformation. Statistical significance was determined by $p$-values $<0.05$.

\section{Results}

Significant correlations were found between peak aspergillus index and chest radiographic stage and between peak eosinophil count and chest radiographic stage among the group as a whole (table 2 and fig. 1a) and these same correlations were higher for the group considered to have "definite" ABPA when considered as a separate subgroup (table 2 and fig. 1). Correlations for the same parameters tended towards, but did not reach significance in the group with "likely" ABPA (table 2 and fig. 1a). Total serum IgE did not correlate with chest radiographic stage.

\section{Discussion}

A wide variety of described chest radiographic changes are associated with ABPA. These changes include normal chest radiography, hyperinflation, various infiltrate patterns, consolidation, parallel line and ring shadows, nodules, avascular areas, "honeycombing", "toothpaste" shadows, "gloved finger", band shadows and tramline shadows. Changes like "fibrosing alveolitis", lobar shrinkage and atelectasis, as well as pseudohilar adenopathy and pleural thickening have also been described [11-16]. No radiographic staging system has been widely accepted for use in this disease. Previous data failed to show any correlation between the immunological findings on the one hand and clinical findings, such as age, duration of asthma or aspergillosis, but were able to demonstrate significant

Table 2. - Correlation of peak aspergillus indices and eosinophil counts with chest radiographic grade

\begin{tabular}{lccllll}
\hline & \multicolumn{2}{c}{$\begin{array}{c}\text { Aspergillus } \\
\text { index }\end{array}$} & & \multicolumn{2}{c}{$\begin{array}{c}\text { Eosinophil } \\
\text { count }\end{array}$} \\
\cline { 2 - 3 } \cline { 6 - 7 } & $\mathrm{r}$ & $\mathrm{p}$ & & $\mathrm{r}$ & $\mathrm{p}$ \\
\hline Whole group $(\mathrm{n}=41)$ & 0.39 & 0.009 & & 0.38 & 0.016 \\
"Definite" ABPA $(\mathrm{n}=13)$ & 0.59 & 0.03 & & 0.62 & 0.02 \\
"Likely" ABPA $(\mathrm{n}=28)$ & 0.33 & 0.08 & & 0.3 & 0.12
\end{tabular}

ABPA: allergic bronchopulmonary aspergillosis. 

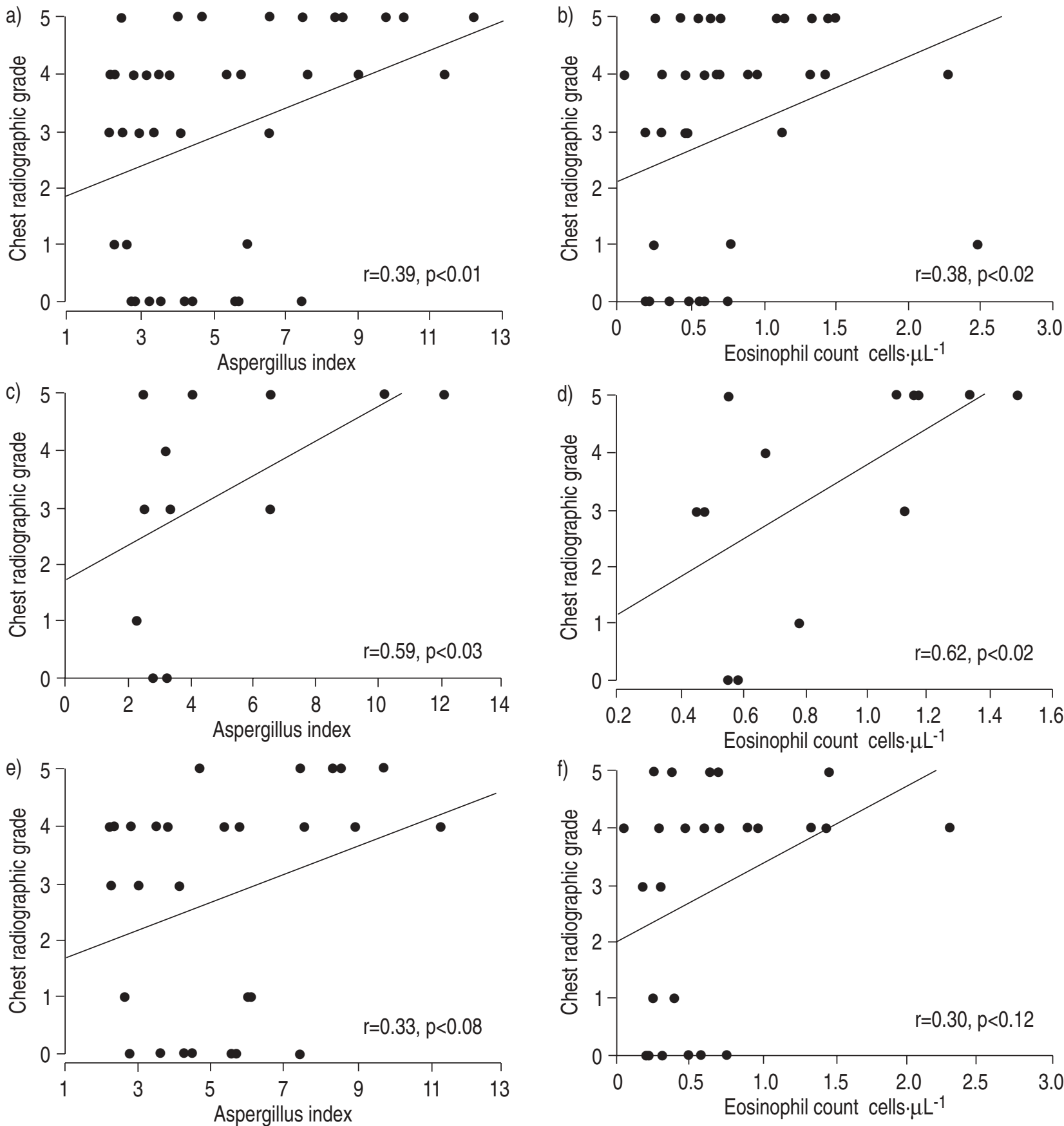

Fig. 1. - Correlations of a) aspergillus index and b) eosinophil count with chest radiographic grade in the whole group. Correlations of c) aspergillus index and d) eosinophil count with chest radiographic grade in "definite" allergic bronchopulmonary aspergillosis (ABPA). Correlations of e) aspergillus index and f) eosinophil count with chest radiographic grade in "likely" ABPA.

relationships between the aspergillus precipitin tests and the radiologically assessed activity of the disease. In the same study [17] IgE levels were not found to have a linear correlation with radiographic changes. These data support the present findings and suggest that AF antibody levels are more closely related than IgE to changes in chest radiographic images.

One would expect higher aspergillus indices and higher degrees of eosinophilia during periods of increased symptomatology or flare-ups to coincide with temporary changes on chest radiography. However, as the current methodology disassociated the highest grades on chest ra- diographs from the blood/serum indices, the relationship between these variables may be due to the reasoning that, over time, patients with flare-ups will eventually progress to some degree of pulmonary scarring. This may appear on the chest radiograph and therefore cause this association.

The present data indicate that the group with "definite" ABPA by our criteria have a significant correlation between both the AF index and chest radiographic stage, and between eosinophil count and chest radiographic stage. The trend in the group with "likely" ABPA, although not reaching statistical significance, is generally in keeping with that of the "definite" group and supports the link 
between the severity of radiographic changes and higher AF indices and eosinophil counts.

A chest radiographic staging system such as the above, together with peak Aspergillus fumigatus indices and eosinophil counts, may be useful in delineating the activity of the disease and, in addition, may help to predict the likelihood of progression of the disease. Accordingly, this staging system may also help in the clinical assessment and management of allergic bronchopulmonary aspergillosis, particularly in those patients with more severe chest radiographic stages.

\section{References}

1. Hinson KFW, Moon AJ, Plummer NS. Bronchopulmonary aspergillosis. Thorax 1952; 7: 317-333.

2. Malo JL, Paquin R. Incidence of immediate sensitivity to Aspergillus fumigatus in a North American asthmatic population. Clin Allergy 1979; 9: 377-384.

3. Hendrick DJ, Ellithorpe DB, Lyon F, Hattier P, Salvaggio JE. Allergic bronchopulmonary helminthosporiosis. Am Rev Respir Dis 1982; 126: 935-938.

4. Halwig JM, Brueske DA, Greenberger PA, Dreisin RB, Sommers HM. Allergic bronchopulmonary curvulariosis. Am Rev Respir Dis 1985; 132: 186-188.

5. Henderson AH, English MP, Vecht RJ. Pulmonary aspergillosis. A survey of its occurrence in patients with chronic lung disease and a discussion of the significance of diagnostic tests. Thorax 1968; 23: 513-518.

6. Donnelly SC, McLaughlin H, Bredin CP. Period prevalence of allergic bronchopulmonary mycosis in a regional hospital outpatient population in Ireland 1985-88: Ir $J$ Med Sci 1991; 160: 288-290.

7. Rosenberg M, Patterson R, Mintzer R, Cooper BJ, Roberts M, Harris KE. Clinical and immunologic criteria for the diagnosis of allergic bronchopulmonary aspergillosis. Ann Intern Med 1977; 86: 405-414.

8. Safirstein BH, D'Sousa MF, Simon G. Five year follow up of allergic broncho-pulmonary aspergillosis. Am Rev Respir Dis 1973; 108: 450-459.

9. Seaton A, Seaton D, Leitch AG. Sarcoidosis. In: Crofton \& Douglas's Respiratory Diseases. Oxford, Blackwell, 1989; pp. 639-643.

10. Patterson R, Greenberger PA, Radin RC, Roberts M. Allergic bronchopulmonary aspergillosis: staging as an aid to management. Ann Intern Med 1982; 96: 286-291.

11. McCarthy DS, Simon G, Hargreave FE. The radiological appearances in allergic broncho-pulmonary aspergillosis. Clin Radiol 1970; 21: 366-375.

12. Gefter WB, Epstein DM, Miller WT. Allergic bronchopulmonary aspergillosis: less common patterns. Diagn Radiol 1981; 140: 307-312.

13. Malo JL, Pepys J, Simon G. Studies in chronic allergic bronchopulmonary aspergillosis. 2. Radiological findings. Thorax 1977; 32: 262-268.

14. Mintzer RA, Rogers LF, Kruglik GD, Rosenberg M, Neiman HL, Patterson R. The spectrum of radiologic findings in allergic bronchopulmonary aspergillosis. Diagn Radiol 1978; 127: 301-307.

15. Angus RM, Davies M-L, Cowan MD, McSharry C, Thomson NC. Computed tomographic scanning of the lung in patients with allergic bronchopulmonary aspergillosis and in asthmatic patients with a positive skin test to Aspergillus fumigatus. Thorax 1994; 49: 586-589.

16. Phelan MS, Kerr IH. Allergic broncho-pulmonary aspergillosis: the radiological appearance during long-term follow-up. Clin Radiol 1984; 35: 385-392.

17. Malo JL, Longbottom J, Mitchell J, Hawkins R, Pepys J. Studies in chronic allergic bronchopulmonary aspergillosis. 3. Immunological findings. Thorax 1977; 32: 269-274. 